\title{
The Anthropometric Assessment With the Bioimpedance Method Is Associated With the Prognosis of Cirrhotic Patients
}

\author{
EI-ICHIRO MORIWAKI ${ }^{1,2}$, HIRAYUKI ENOMOTO ${ }^{1}$, MASAKI SAITO $^{1}$, NAOKI HARA ${ }^{2}$, HIROKI NISHIKAWA ${ }^{1}$, \\ TAKASHI NISHIMURA ${ }^{1}$, YOSHINORI IWATA ${ }^{1}$, HIROKO IIJIMA ${ }^{1}$ and SHUHEI NISHIGUCHI ${ }^{1}$ \\ ${ }^{1}$ Division of Hepatobiliary and Pancreatic Disease, \\ Department of Internal Medicine, Hyogo College of Medicine, Hyogo, Japan; \\ ${ }^{2}$ Department of Gastroenterology, Kano General Hospital, Osaka, Japan
}

\begin{abstract}
Background/Aim: The bioimpedance analysis (BIA) can provide anthropometric data on patients. The aim of the study is to evaluate the clinical relevance of these automatically obtained values. Patients and Methods: We studied the arm circumference (AC) and arm muscle circumference (AMC) of 197 histologically proven cirrhotic patients. The BIA-based anthropometric data were compared to the manually measured data. In addition, we evaluated whether or not the BIA-based anthropometric data were associated with the prognosis of the patients. Results: The data of \%AC and \%AMC obtained using the two methods were well correlated $(p<0.001)$ with relatively inconspicuous differences (approximately $6.0 \%$ for $\% A C$ and $16.0 \%$ for $\% A M C)$. The data of \%AC and \%AMC obtained from the BIA method were significantly associated with the prognosis of the patients. Conclusion: The BIA-based anthropometric data were associated with the direct measurement data and related to the prognosis of cirrhotic patients.
\end{abstract}

Patients with liver cirrhosis frequently suffer from proteinenergy malnutrition (PEM) and sarcopenia, and the severity of these complications is associated with their prognosis $(1,2)$. Anthropometric measurement is a useful method for noninvasively assessing the nutritious status of patients using only a tape measure, and some variables, such as the arm circumference (AC) or the arm muscle circumference (AMC),

This article is freely accessible online.

Correspondence to: Hirayuki Enomoto, Division of Hepatobiliary and Pancreatic Disease, Department of Internal Medicine, Hyogo College of Medicine, Mukogawa-cho1-1, Nishinomiya, Hyogo 6638501, Japan. Tel: +81 798456472, Fax: +81 798456474, e-mail: enomoto@hyo-med.ac.jp

Key Words: Bioimpedance analysis, arm circumference, arm muscle circumference, liver cirrhosis, prognosis. can reflect the nutritional status without using any special equipment $(3,4)$. Although assessment of the nutrition based on anthropometric measurements has been reported to show fair to good reproducibility (5), such manual measurements may result in some intra-measurer discrepancies. However, it is difficult to establish an optimal system for evaluating all patients by sufficiently qualified persons in daily clinical practice.

According to Asian guidelines, including the Japanese guideline regarding sarcopenia in patients with chronic liver diseases (6-8), the bioimpedance analysis (BIA) is one option for objectively assessing the muscle mass of patients. In the BIA method, the estimated values of AC and AMC can be automatically displayed; however, precisely how the values are calculated remains a manufacturer's secret, and whether or not the automatically obtained values accurately reflect the manually measured physical data is unclear.

We assessed the nutritional status of cirrhotic patients using various methods (9-11), and a dietitian in our department took anthropometric measurements from all patients. In this study, we evaluated the nutritional status of histologically confirmed cirrhotic patients (F4 stage of the METAVIR scoring system: liver cirrhosis with pseudolobules) (12) and compared the physical data automatically provided with the BIA method to the manually obtained ones by a trained healthcare professional. In addition, we assessed whether or not the automatically available data reflected the prognosis of patients.

\section{Patients and Methods}

Liver biopsy and blood sample collection. Percutaneous liver biopsy examinations were performed via ultrasound-guided standard procedures. The stage of liver fibrosis was determined according to the METAVIR scoring system (12): i) F0, no fibrosis, ii) F1 as portal fibrosis without septa, iii) F2 as portal fibrosis with rare septa, iv) F3 as fibrosis with numerous septa but without cirrhosis, and v) F4 as liver cirrhosis with pseudo-lobules. All medical data, including basic clinical variables, were collected under fasting conditions on the same day as the liver biopsies. 
Table I. Characteristics of enrolled patients.

\begin{tabular}{lc}
\hline Age (years) & $68(25-90)$ \\
Gender (Male/Female) & $117 / 80$ \\
Child-Pugh grade (A/B/C) & $122 / 67 / 8$ \\
Etiology (HBV/HCV/HBV+ & \\
HCV/ALD NASH/AIH PBC/Others) & $14 / 126 / 2 / 21 / 6 / 10 / 6 / 12$ \\
Body Mass Index (Kg/m $\left.{ }^{2}\right)$ & $22.2(13.6-38.7)$ \\
AST $(\mathrm{IU} / \mathrm{l})$ & $46(16-577)$ \\
ALT $(\mathrm{IU} / \mathrm{l})$ & $33(9-437)$ \\
$\gamma$-GTP $(\mathrm{IU} / \mathrm{l})$ & $43(10-901)$ \\
ALP $(\mathrm{IU} / \mathrm{l})$ & $320(112-2193)$ \\
Total bilirubin $(\mathrm{mg} / \mathrm{dl})$ & $0.9(0.2-12.3)$ \\
Albumin $(\mathrm{g} / \mathrm{dl})$ & $3.6(2.4-4.9)$ \\
Hemoglobin $(\mathrm{g} / \mathrm{dl})$ & $12.0(6.6-17.5)$ \\
Platelet count $\left(\times 10^{3 / \mu l}\right)$ & $91(24-428)$ \\
Prothrombin time $(\%)$ & $77.0(30.2-115.6)$ \\
Glucose $(\mathrm{mg} / \mathrm{dl})$ & $101(59-296)$ \\
Total cholesterol $(\mathrm{mg} / \mathrm{dl})$ & $150(73-292)$ \\
Triglyceride & $75(30-373)$ \\
\hline
\end{tabular}

HBV: Hepatitis B virus; HCV: hepatitis C virus; ALD: alcoholic-related liver disease; NASH: nonalcoholic steatohepatitis; AIH; autoimmune hepatitis; PBC: primary biliary cholangitis; IU: international unit.

The study conformed to the ethical guidelines of the Declaration of Helsinki (13) and was approved by the ethics committee of the institutional review board (Nos. Rin-Hi-92 and 1831). Written informed consent with regard to the use of clinical data was obtained from all patients before the liver biopsy.

Determination of $\% A C$ and $\% A M C$. In the present study, we evaluated a total of 197 patients who received a liver biopsy from February 2006 to January 2012 and were histologically diagnosed as having cirrhosis (F4). The nutritious status of the patients was assessed via both manual anthropometric measurement and the BIA method on the same day as the liver biopsy. Regarding the manually obtained data, a designated dietitian directly measured the values of the $\mathrm{AC}(\mathrm{cm})$ and the triceps skinfold thickness (TSF) $(\mathrm{cm})$. The AMC values were obtained using the following formula:

$\operatorname{AMC}(\mathrm{cm})=\operatorname{AC}(\mathrm{cm})-\pi \cdot \mathrm{TSF}(\mathrm{cm})$

The body composition analyzer InBody $720^{\circledR}$ (Inbody Japan, Tokyo, Japan), was used for the BIA method in the current study. The values of \% AC and \% AMC were determined with reference to the standard values of AC and AMC in Japanese people (14).

Statistical analyses. The relationship between the data obtained manually and those obtained via the BIA method were determined using the Spearman's correlation coefficient. We categorized the quantitative parameters into two groups according to the median value of each parameter, and the differences in the paired data between two groups were evaluated with Wilcoxon's signed-rank test. Regarding the relationship between the nutritional data and the prognosis, survival curves were generated using the Kaplan-Meier method and compared using the log-rank test. A $p$-value $<0.05$ was considered significant.
Table II. Comparison the anthropometric data between the manually measured and the BIA method.

\begin{tabular}{lcccc}
\hline & $\begin{array}{c}\text { Manual measurement } \\
(\mathrm{cm})\end{array}$ & $\begin{array}{c}\text { BIA method } \\
(\mathrm{cm})\end{array}$ & $\begin{array}{c}\text { Difference } \\
(\mathrm{cm})\end{array}$ & $p$-Value \\
\hline AC & $26.7(18.9-37.8)$ & $28.2(12.2-40.9)$ & $1.51 \pm 0.13$ & $<0.01$ \\
AMC & $21.2(13.6-31.3)$ & $24.8(18.8-33.3)$ & $3.26 \pm 0.16$ & $<0.01$ \\
\hline
\end{tabular}

AC: Arm circumference; AMC: arm-muscle circumference.

\section{Results}

Basic characteristics of the histologically proven cirrhotic patients. The survival of a total of 197 patients was retrospectively analyzed, and the mean follow-up period was around 4.2 years (1527.9 days). The basic clinical characteristics of the enrolled patients are shown in Table I. Among the 197 patients, 117 (59.3\%) were men, and the age ranged from 25 to 90 (median $=68$ years old). The total number of hepatitis virus-related cirrhosis cases was 142 $(72.1 \%)$, and that of non-viral cirrhosis was 55 (27.9\%).

The comparison of the automatically calculated physical data to the data obtained via manual measurement. In the present study, we focused on two parameters (AC and AMC) and compared the results measured by the BIA method with those manually measured by a designated dietitian (Table II). Because of the large number of data $(\mathrm{N}=197)$, the BIA method provided significantly higher values of $\mathrm{AC}$ and AMC compared to the manually measured data; however, the differences in the values of $\mathrm{AC}$ and $\mathrm{AMC}$ between the two methods were relatively small (mean differences $=1.51 \mathrm{~cm}$ and $3.26 \mathrm{~cm}$, respectively) (Table II). When we calculated the values of $\% \mathrm{AC}$ and \%AMC, the BIA-based data were significantly higher compared to the manually measured data both for $\% \mathrm{AC}$ (Figure 1A) and \%AMC (Figure 1B). However, the differences between the BIA data and the directly measured data were also relatively inconspicuous (approximately 6.0\% for $\% \mathrm{AC}$ and $16.0 \%$ for $\% \mathrm{AMC}$ ) (Figure 1C), and the data from the two methods were significantly correlated for the AC, \%AC, AMC and \%AMC values (Figures 2 and 3).

The prognosis of cirrhotic patients in relation to the physical data. We evaluated the prognosis of cirrhotic patients according to the manually obtained anthropometric values of $\%$ AC and \%AMC (Figure 4). As predicted, the high-value group of $\% \mathrm{AC}$ had a significantly better prognosis compared to the low-value group ( $p=0.0239$ ). The high-value group of $\%$ AMC also showed a significantly better prognosis compared to the low-value group $(p=0.0084)$. When we evaluated the data of \% AC and \%AMC that had been automatically 
A

(\%)

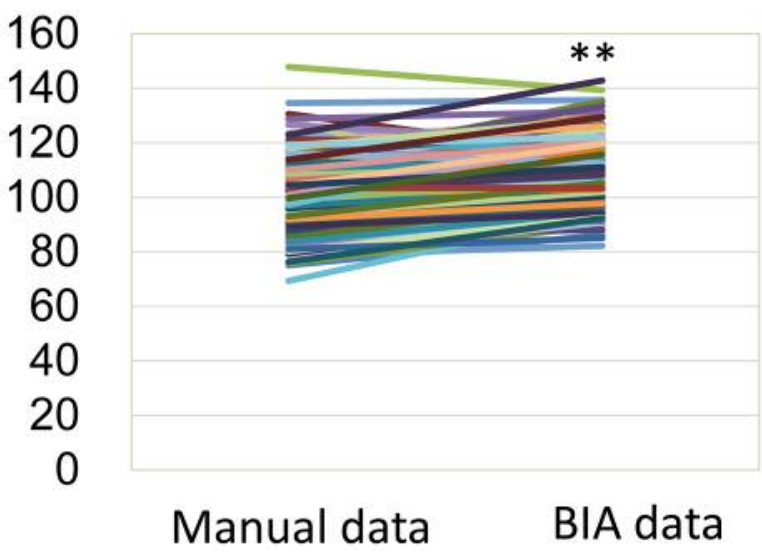

(\%)

160

140

120

100

80

60

40

20

0

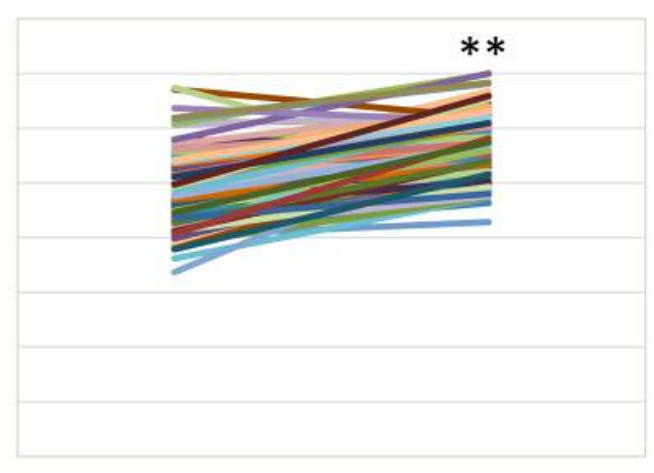

Manual data BIA data

(\%)

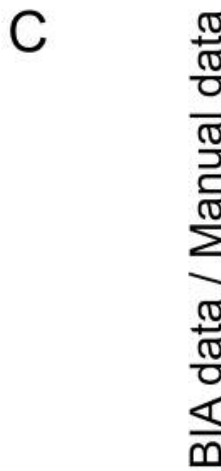

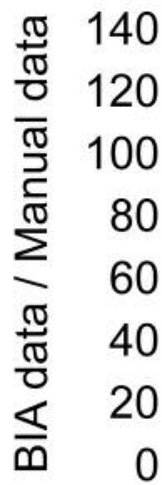

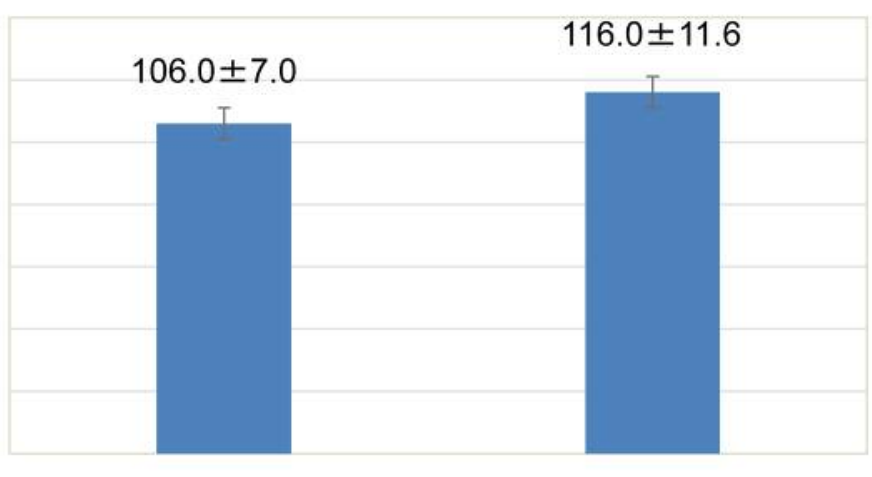

$\% A C$

$\% A M C$

Figure 1. Comparing the \%AC and \%AMC values between the BIA-based data and the manually obtained data. The \%AC and \%AMC data were calculated with reference to the Japanese anthropometric data (15). (A) The \%AC data obtained using the BIA and manual measurements are shown. (B) The \%AMC data obtained using the BIA and manual measurements are shown. Due to the large number of enrolled patients (N=197), the values obtained with the BIA method were significantly higher compared to those obtained via manual measurements $(* * p<0.01)$. (C) The ratio of the BIA-based data to the manually measured data is shown. The discrepancy in the data between the two methods was not marked (approximately $6.0 \%$ for \%AC and $16.0 \%$ for \%AMC). AC: Arm circumference; AMC: arm muscle circumference; BIA: bioimpedance analysis.

obtained by the BIA method (Figure 5), the patients with high $\% \mathrm{AC}$ values had a significantly higher survival rate compared to those with low \%AC values $(p=0.0358)$. In addition, the high \%AMC group had a favorable prognosis as compared to the low \%AMC group $(p=0.0362)$.

\section{Discussion}

It has been reported that PEM and sarcopenia are related to the prognosis of patients with cirrhosis. In the Japanese guideline for the management of liver cirrhosis (3), measuring the non-protein respiratory quotient (npRQ) with an indirect calorimeter (15) is described as the gold-standard method for assessing energy malnutrition. However, the indirect calorimeter method requires special equipment and cannot be easily performed in all patients. The Japanese guideline for the management of liver cirrhosis mentions that the \% AC value can be used as a simple indicator of energy malnutrition substituting the npRQ measurement, since the two are linked and a low \% AC is associated with a decrease in the npRQ (3). A reduced muscle mass, including a decreased \% AMC, is also known to be associated with an unfavorable clinical course in 

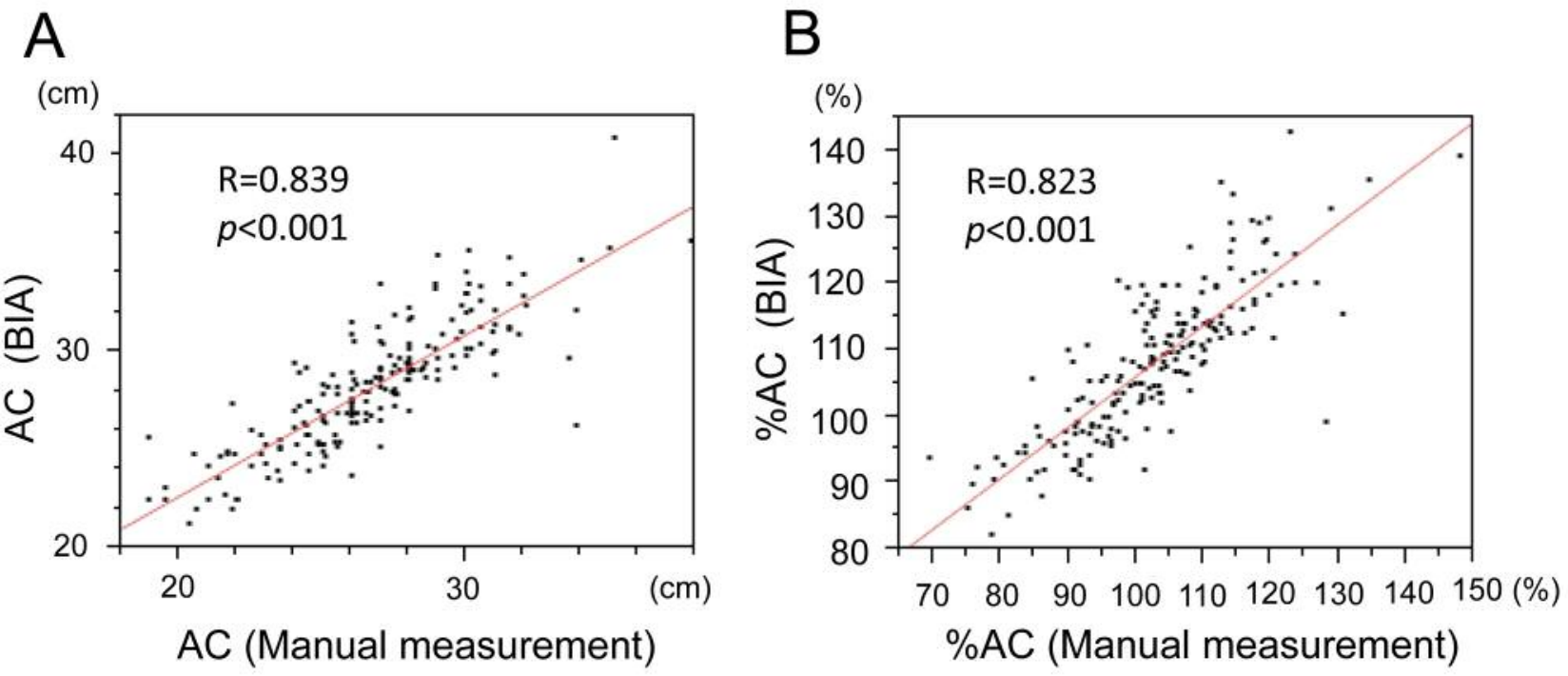

Figure 2. Relationships of the AC and \%AC between the BIA-based data and the manually obtained data. (A) Relationships of the AC values between the BIA-based data and the manually obtained data. (B) Relationships of the \%AC values between the BIA-based data and the manually obtained data. The anthropometric values obtained using the BIA-based method were significantly associated with those obtained using manual measurements. AC: Arm circumference; BIA: bioimpedance analysis.
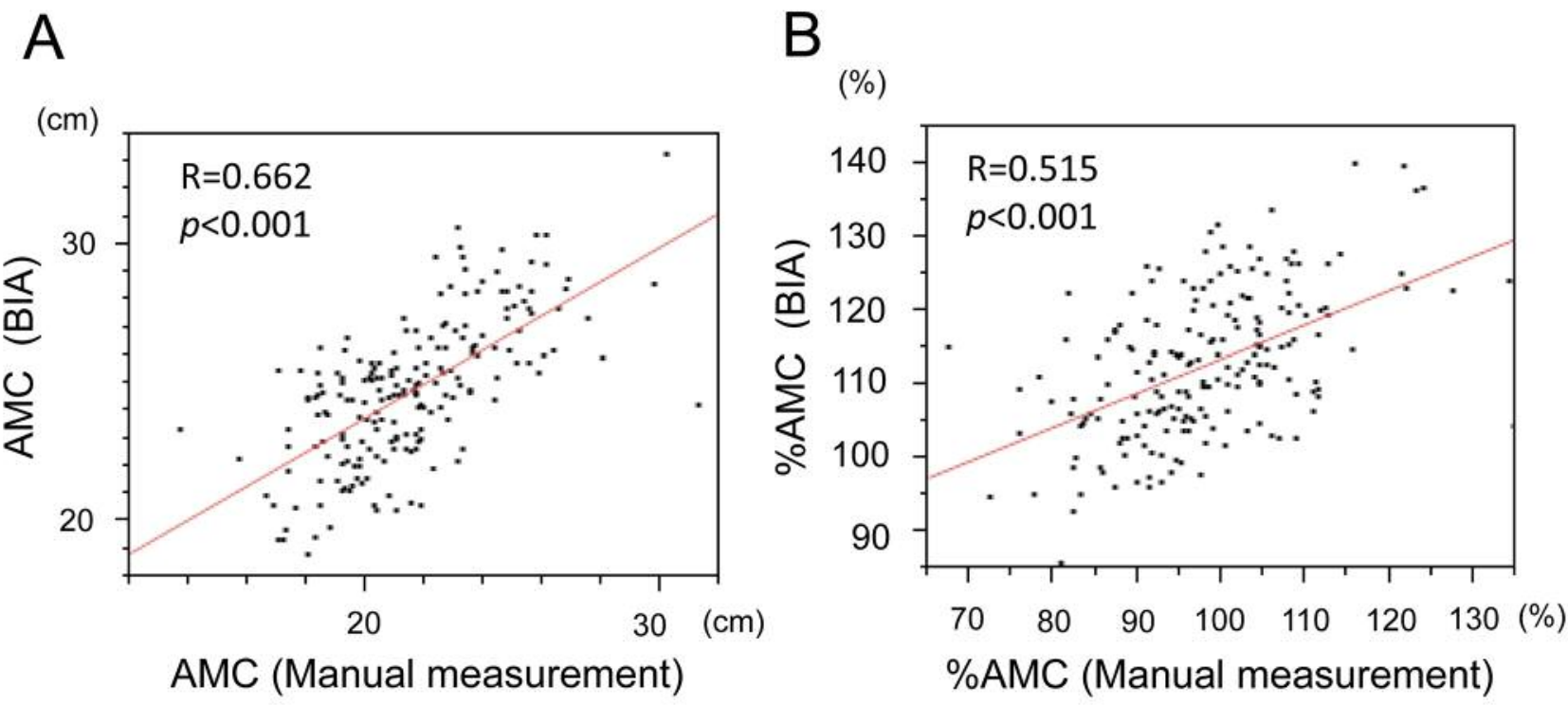

Figure 3. Relationships of the AMC and \%AMC values between the BIA-based data and the manually obtained data. (A) Relationships of the AMC values between the BIA-based data and the manually obtained data. (B) Relationships of the \%AMC values between the BIA-based data and the manually obtained data. The anthropometric values obtained by the BIA-based method were significantly associated with those obtained by manual measurements. AMC: Arm muscle circumference; BIA: bioimpedance analysis.

cirrhotic patients (16-20). In the present study, we showed that the physical data automatically estimated with the BIA method correlated well with the directly measured physical data and were related to the prognosis of the cirrhotic patients as well the directly measured anthropometric data.
The anthropometric measurement is a simple method that requires no special resources. However, this noninvasive and inexpensive method requires a certain amount of time and effort, particularly for evaluating a large number of patients. In addition, because of the risk of intra-measurer discrepancy, 

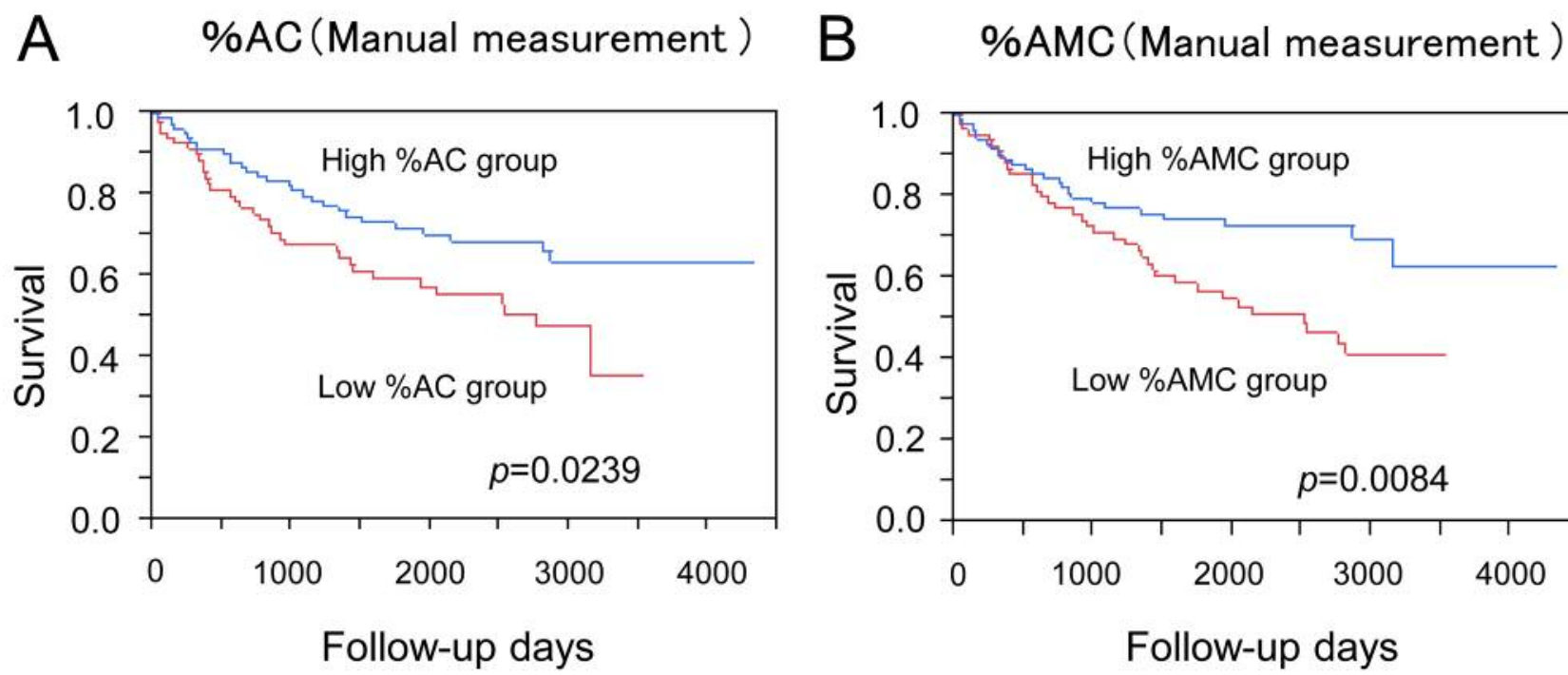

Figure 4. Comparing the survival curves according to the manually obtained data for \%AC and \%AMC. (A) Patients with high \%AC values showed a significantly better survival compared to those with low \%AC values. (B) Patients with high \%AMC values showed a significantly better survival compared to those with low \%AMC values. AC: Arm circumference; AMC: arm muscle circumference.

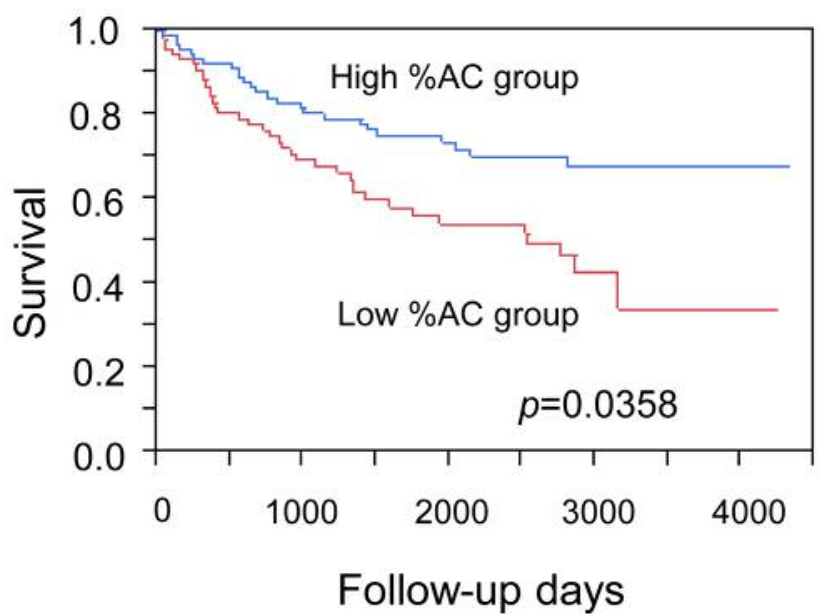

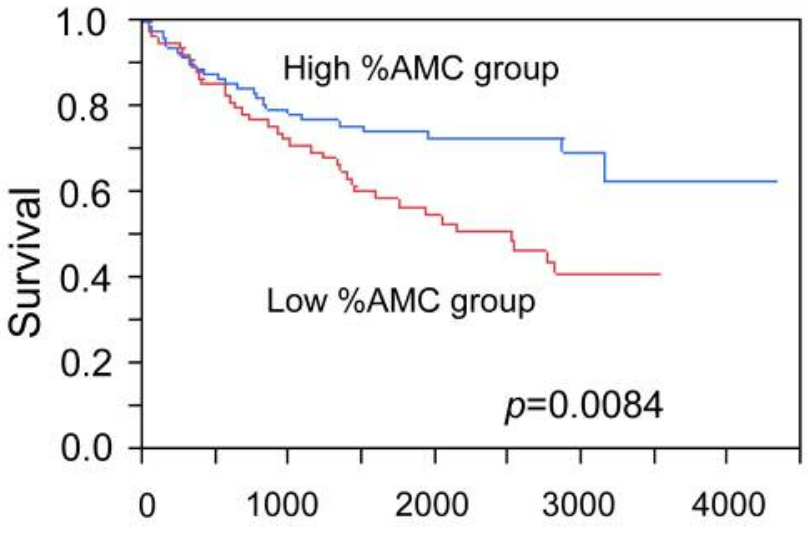

Follow-up days

\section{A $\quad$ \%AC (BIA) B}

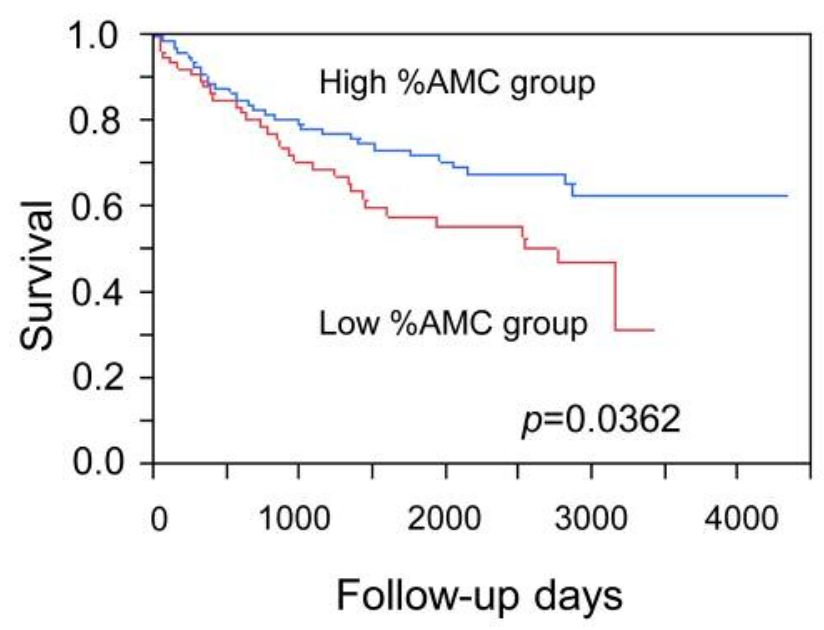

Figure 5. Comparing the survival curves according to the BIA-based data for \%AC and \%AMC. (A) Similar to the manually obtained data (Figure $4 A)$, patients with high \%AC values had a significantly better survival compared to those with low \%AC values. (B) Similar to the manually obtained data (Figure 4B), patients with high \%AMC values showed a significantly better survival compared to those with low \%AMC values. BIA: Bioimpedance analysis; AC: arm circumference; AMC: arm muscle circumference.

stable measurement standards carried out by skilled personnel are desired to achieve accurate evaluations. Therefore, in our institution, a single dedicated dietitian has been collecting the data of all patients. However, ideally, a tool should be developed that can be easily used even by an untrained person and provide reasonable diagnostic accuracy $(2,21,22)$.
The physical data collected using the BIA method can be automatically obtained with little human burden even in the absence of a trained healthcare expert. This is the first study to compare manually measured physical data to BIA-based data. In addition, the current study is unique in that the BIAbased automatically obtained data for \% AC and \% AMC 
were shown to be relevant for the prognosis of cirrhotic patients. Despite the advantage of our accumulated data being obtained by a designated skilled dietitian, we found that the automatically available data correlated well with the directly measured data and were associated with the prognosis of cirrhotic patients. Our findings suggested that the physical data obtained with the BIA method are thus comparable to those obtained by a trained person for anthropometric measurements and can be clinically useful.

Several limitations associated with the present study warrant mention. First, our study included all cirrhotic patients, and their nutritious status may have differed depending on the etiologies. Second, the study enrolled histologically proven cirrhotic patients (F4 stage), and the number of patients was relatively small. Finally, our directly measured anthropometric data were obtained by a designated dietitian. The manually determined values of AC and AMC were low compared to those determined via the BIA method, although this discrepancy may simply have been due to the standards set by the measurer. However, the muscle volume estimated by the BIA method may have been influenced by the fluid collection. Cirrhotic patients are known to have a tendency to store fluid throughout their body $(11,23)$; such cirrhosis-associated clinical characteristics may therefore have influenced the discrepancy in the data between the manual measurement and the BIA method. We showed the clinical relevance of the BIA method when the data were analyzed; however, we should take care not to combine anthropometric data obtained using different methods.

In conclusion, we investigated the relationship of the physical data obtained using the BIA method with those directly measured in patients with histologically confirmed cirrhosis using liver biopsy. The anthropometric data obtained using the BIA method, particularly the AC and $\%$ AC values, were well associated with those obtained using direct measurement and were found to be related to the prognosis of cirrhotic patients.

\section{Conflicts of Interest}

The Authors declare no conflicts of interest.

\section{Authors' Contribution}

EM collected and analyzed the data and HE designed the study, analyzed the data and wrote the manuscript. MS, NH, HN, TN, YI and $\mathrm{HI}$ collected the data, SN designed the study and analyzed the data. All Authors reviewed and edited the manuscript and approved the final version of the manuscript.

\section{Acknowledgements}

The Authors are grateful to Higuchi Y, Inui S, Kanazawa N, Matsushita Y, Fujii S, Kido H and Minemoto K (Hyogo College of Medicine) for their technical and secretarial assistance.

\section{References}

1 European Association for the Study of the Liver: EASL Clinical Practice Guidelines on nutrition in chronic liver disease. J Hepatol 70(1): 172-193, 2019. PMID: 30144956. DOI: 10.1016/ j.jhep.2018.06.024

2 Tandon P, Raman M, Mourtzakis M and Merli M: A practical approach to nutritional screening and assessment in cirrhosis. Hepatology 65(3): 1044-1057, 2017. PMID: 28027577. DOI: 10.1002/hep.29003

3 Fukui H, Saito H, Ueno Y, Uto H, Obara K, Sakaida I, Shibuya A, Seike M, Nagoshi S, Segawa M, Tsubouchi H, Moriwaki H, Kato A, Hashimoto E, Michitaka K, Murawaki T, Sugano K, Watanabe $\mathrm{M}$ and Shimosegawa T: Evidence-based clinical practice guidelines for liver cirrhosis 2015. J Gastroenterol 51(7): 629-650, 2016. PMID: 27246107. DOI: 10.1007/s00535-016-1216-y

4 Alberino F, Gatta A, Amodio P, Merkel C, Di Pascoli L, Boffo $\mathrm{G}$ and Caregaro L: Nutrition and survival in patients with liver cirrhosis. Nutrition 17(6): 445-450, 2001. PMID: 11399401. DOI: $10.1016 / \mathrm{s} 0899-9007(01) 00521-4$

5 Hasse J, Strong S, Gorman MA and Liepa G: Subjective global assessment: alternative nutrition-assessment technique for livertransplant candidates. Nutrition 9(4): 339-343, 1993. PMID: 8400590.

6 Chen LK, Lee WJ, Peng LN, Liu LK, Arai H, Akishita M; Asian Working Group for Sarcopenia: Recent Advances in Sarcopenia Research in Asia: 2016 Update from the Asian Working Group for Sarcopenia. J Am Med Dir Assoc 17(8): 767.e1-7, 2016. PMID: 27372539. DOI: 10.1016/j.jamda.2016.05.016

7 Xia W, Cooper C, Li M, Xu L, Rizzoli R, Zhu M, Lin H, Beard J, Ding Y, Yu W, Cavalier E, Zhang Z, Kanis JA, Cheng Q, Wang Q and Reginster JY: East meets West: current practices and policies in the management of musculoskeletal aging. Aging Clin Exp Res 31(10): 1351-1373, 2019. PMID: 31376119. DOI: 10.1007/s40520-019-01282-8

8 Nishikawa H, Shiraki M, Hiramatsu A, Moriya K, Hino K and Nishiguchi S: Japan Society of Hepatology guidelines for sarcopenia in liver disease (1st edition): recommendation from the working group for creation of sarcopenia assessment criteria. Hepatol Res 46(10): 951-963, 2016. PMID: 27481650. DOI: 10.1111/hepr.12774

9 Takata R, Nishikawa H, Enomoto H, Iwata Y, Ishii A, Miyamoto Y, Ishii N, Yuri Y, Hasegawa K, Nakano C, Nishimura T, Yoh K, Aizawa N, Sakai Y, Ikeda N, Takashima T, Iijima H and Nishiguchi S: Relationship between skeletal muscle mass and liver fibrosis markers for patients with hepatitis $\mathrm{C}$ virus related liver disease. Medicine (Baltimore) 96(48): e8761, 2017. PMID: 29310350. DOI: 10.1097/MD.0000000000008761

10 Nishikawa H, Enomoto H, Yoh K, Iwata Y, Sakai Y, Kishino K, Ikeda N, Takashima T, Aizawa N, Takata R, Hasegawa K, Ishii $\mathrm{N}$, Yuri Y, Nishimura T, Iijima $\mathrm{H}$ and Nishiguchi S: Effect of Sarcopenia on Sleep Disturbance in Patients with Chronic Liver Diseases. J Clin Med 8(1) pii: E16, 2018. PMID: 30583494. DOI: $10.3390 / \mathrm{jcm} 8010016$

11 Nishikawa H, Enomoto H, Iwata Y, Nishimura T, Iijima H and Nishiguchi S: Clinical utility of bioimpedance analysis in liver cirrhosis. J Hepatobiliary Pancreat Sci 24(7): 409-416, 2017. PMID: 28371518. DOI: $10.1002 /$ jhbp. 455

12 The French METAVIR Cooperative Study Group: Intraobserver and interobserver variations in liver biopsy interpretation in patients with chronic hepatitis C. Hepatology 20(1 Pt 1): 15-20, 1994. PMID: 8020885. 
13 WMA Declaration of Helsinki - Ethical Principles for Medical Research Involving Human Subjects. Adopted by the 18th WMA General Assembly, Helsinki, Finland, June 1964 and amended by the 64th WMA General Assembly, Fortaleza, Brazil, October 2013. Available at https://www.wma.net/policies-post/wmadeclaration-of-helsinki-ethical-principles-for-medical-researchinvolving-human-subjects/Last accessed on 21st November 2019.

14 Hosoya N. Japanese anthropometric reference data 2001 (JARD 2001) Japanese Journal of Nutritional Assessment 19(Suppl): 181, 2002.

15 Tajika M, Kato M, Mohri H, Miwa Y, Kato T, Ohnishi H and Moriwaki H: Prognostic value of energy metabolism in patients with viral liver cirrhosis. Nutrition 18(3): 229-234. 2002. PMID: 11882395. DOI: 10.1016/s0899-9007(01)00754-7

16 Ebadi M, Bhanji RA, Mazurak VC and Montano-Loza AJ: Sarcopenia in cirrhosis: from pathogenesis to interventions. J Gastroenterol 54(10): 845-859, 2019. PMID: 31392488. DOI: 10.1007/s00535-019-01605-6

17 Bhanji RA, Montano-Loza AJ and Watt KD: SARCOPENIA IN CIRRHOSIS: Looking beyond the skeletal muscle loss to see the systemic disease. Hepatology 70(6): 2193-2203, 2019. PMID: 31034656. DOI: 10.1002/hep.30686

18 Palmer LB, Kuftinec G, Pearlman M and Green CH: Nutrition in Cirrhosis. Curr Gastroenterol Rep 21(8): 38, 2019. PMID: 31289936 DOI: $10.1007 / \mathrm{s} 11894-019-0706-5$

19 Vasques J, Guerreiro CS, Sousa J, Pinto M and Cortez-Pinto H: Nutritional support in cirrhotic patients with sarcopenia. Clin Nutr ESPEN 33: 12-17, 2019. PMID: 31451247. DOI: 10.1016/ j.clnesp.2019.07.011
20 Lucidi C, Lattanzi B, Di Gregorio V, Incicco S, D’Ambrosio D, Venditti M, Riggio O and Merli M: A low muscle mass increases mortality in compensated cirrhotic patients with sepsis. Liver Int 38(5): 851-857, 2018. PMID: 29323441. DOI: 10.1111/liv.13691

21 Marr KJ, Shaheen AA, Lam L, Stapleton M, Burak K and Raman M: Nutritional status and the performance of multiple bedside tools for nutrition assessment among patients waiting for liver transplantation: A Canadian experience. Clin Nutr ESPEN 17: 68-74, 2017. PMID: 28361750. DOI: 10.1016/j.clnesp. 2016.10.003

22 Laporte M, Keller HH, Payette H, Allard JP, Duerksen DR, Bernier P, Jeejeebhoy K, Gramlich L, Davidson B, Vesnaver E and Teterina A: Validity and reliability of the new Canadian Nutrition Screening Tool in the 'real-world' hospital setting. Eur J Clin Nutr 69(5): 558-564, 2015. PMID: 25514899. DOI: 10.1038/ejen.2014.270

23 Montano-Loza AJ: Clinical relevance of sarcopenia in patients with cirrhosis. World J Gastroenterol 20(25): 8061-8071, 2014. PMID: 25009378. DOI: 10.3748/wjg.v20.i25.8061
Received November 23, 2019

Revised December 3, 2019

Accepted December 10, 2019 